\title{
SUN13837 in Treatment of Acute Spinal Cord Injury, the ASCENT-ASCI Study
}

\author{
Benjamin Levinson ${ }^{1, ~ *}$, James Lee ${ }^{1}$, Hubert Chou ${ }^{1}$, Dennis Maiman ${ }^{2}$ \\ ${ }^{1}$ Department of Clinical Research, Daiichi-Sankyo Pharma Development, Basking Ridge, USA \\ ${ }^{2}$ Department of Neurosurgery, Medical College of Wisconsin, Milwaukee, USA \\ Email address: \\ blevinson@dsi.com (B. Levinson) \\ ${ }^{*}$ Corresponding author
}

\section{To cite this article:}

Benjamin Levinson, James Lee, Hubert Chou, Dennis Maiman. SUN13837 in Treatment of Acute Spinal Cord Injury, the ASCENT-ASCI Study. Clinical Neurology and Neuroscience. Vol. 2, No. 1, 2018, pp. 1-8. doi: 10.11648/j.cnn.20180201.11

Received: October 26, 2017; Accepted: November 8, 2017; Published: December 23, 2017

\begin{abstract}
The purpose of this study was to assess the safety and efficacy of SUN13837, a bFGF mimetic, for the treatment of acute cervical spinal cord injury. In a 26-week, double-blind trial, 65 subjects were randomized (1:1) by stratum within 12 hours of injury to $1 \mathrm{mg} / \mathrm{kg}$ /day of intravenous SUN13837 (SUN) or matching placebo (pbo) for no less than 7 and no more 28 days. The efficacy measures at Week 16 were the mean total SCIM III (primary), combined SCIM III Self-Care and Mobility subscales (secondary) and ISNCSCI Total Motor scores (secondary). Of the 61 subjects who received study drug, 57 received at least 7 doses. 55 subjects (ITT population) were assessed by treatment assignment and by strata of C4-C5 AIS A (pbo=13, $\mathrm{SUN}=15)$, C6-C7 AIS A (pbo=6, SUN=5) or C3-C8 AIS B and C (pbo=9, SUN=7). The majority of subjects were Male $(85.2 \%)$, Caucasian (63.9\%). The Total SCIM III score between the 2 treatment groups at Week 16 was 4.54 (SE $=6.524)$, not statistically significant with $\mathrm{p}=0.4912$. Therefore, the primary end-point was not reached. Overall, larger effects were observed in AIS C6-C7 and AIS B and C strata as compared with AIS A C4-C5. Specifically, in the C3-8 AIS B and C stratum, a 6.8-point difference (LS) in Total SCIM III was observed (SUN vs. pbo). However, there were more AIS C subjects in the SUN ( $n=5)$ vs. pbo $(n=2)$. The combination of self-care and mobility scores was not statistically significant with p-value $=0.3951$. By Week 16, the LS Mean (SE) change from baseline in UEMS scores was 9.92 in SUN13837-treated subjects compared to 4.95 in Placebo-treated subjects ( $p$-value $=0.0347$ ). The largest treatment difference was seen in the AIS B and C strata in which SUN13837-treated subjects had an average change from baseline in UEMS of 25.40 compared to 6.86 in Placebo-treated subjects. As a result, the AIS B and C stratum may have contributed heavily to the overall treatment effect on the UEMS with lesser contribution by AIS A C4-C5 and C6-C7 strata. Analyses of primary and secondary outcomes showed non-significant trends consistently favoring SUN13837 treatment. The efficacy signal of SUN13837 warrants further investigation. No safety concerns were noted by an Independent Data Safety Monitoring and Review Board. Pharmacokinetic modeling indicates that the dose may need to be lowered in any further evaluation of SUN13837 (NCT01502631).
\end{abstract}

Keywords: SUN13837, Spinal Cord Injury, Neuroprotection, bFGF

\section{Introduction}

Acute spinal cord injury (ASCI) often leads to substantial disability due to permanent neurological impairment if the patient survives the injury. The annual incidence of ASCI in the US is about 13,200 to 15,600 cases per year [1] and well over $50 \%$ of cases have some degree of tetraplegia. The life expectancy of patients with ASCI is dramatically and progressively shortened ${ }^{1}$ in relation to the level of the spinal cord injury and the degree of neurological deficit. The estimated lifetime costs for 25-year-old patients with tetraplegia range between 1.7 and 3.1 million dollars and for patients with paraplegia, around 1 million dollars [2].

Although high dose steroids are commonly used, there is currently no drug treatment approved by US or European Union regulatory agencies for ASCI. Acute spinal cord injury is a catastrophic medical condition which dramatically reduces the patient's quality of life and imposes disproportionately large economic and social costs on affected individuals and society in general $[3,4]$. 
The term ASCI denotes a process that begins with an external insult to the integrity of the neural pathways of the spinal cord and culminates in an anatomical lesion from which a myriad of physical incapacities result. The mechanism that leads to the anatomical lesion and its evolution over time has been the focus of intense study. Research has uncovered several pathophysiological events that have been explored with various degrees of nonclinical and clinical success.

Basic fibroblast growth factor has shown neuroprotective and neuroregenerative properties, which could reduce neuronal damage and improve recovery following ASCI [5]. However, bFGF possesses other undesirable physiological effects, such as limited penetration through an intact blood brain barrier due to its macromolecular size [6] and cell proliferative effects [7] that limit its potential as a therapeutic agent in ASCI.

In contrast, SUN13837 is a small lipid soluble molecule making it more likely to penetrate the blood brain barrier. SUN13837, 2-([5-Amino-4, 6-dimethylpyrimidin-2-yl] oxy)$\mathrm{N}$-(1-benzylpiperidin-4-yl)-N-methylacetamide, is a novel small molecule targeted as a potential treatment for ASCI. SUN13837 exerts various biological activities similar to those of bFGF through modulation of the signal transduction pathway of the fibroblast growth factor receptor; however, SUN13837 does not possess the same proliferative effects on the cell as bFGF.

Once daily intravenous dosing of $1.0 \mathrm{mg} / \mathrm{kg}$ study drug was chosen for this study based on nonclinical pharmacology studies, which showed that the SUN13837 may act as an agonist of fibroblast growth factor receptors and once a day treatment should be enough for maximum efficacy. The PK profile of SUN13837 in healthy subjects supported once a day dosing.

The choice of 28 days of dosing was based on the theoretical concern that the post-traumatic neurological spinal cord scar formation (glial scar formation) would be complete by this time point preventing further drug penetration afterwards. However, during the course of the study, additional nonclinical data supported a dosing reduction to at least 7 (partial or complete) IV bolus dose injections.

\section{Methods}

\subsection{Study Design}

This was a phase 2, multicenter, randomized, double-blind, Placebo-controlled, parallel group study. The purpose of this phase 2 study was to provide the necessary efficacy, safety, and pharmacokinetic (PK) data to support further development of SUN13837 injection in a broad population of patients with ASCI. The study was conducted in compliance with ethical principles that have their origin in the Declarations of Helsinki and in accordance with the following: 1) US Code of Federal Regulations (CFR) Title 21, parts 11, 50, 54, 56 and 312 2); US Code of Federal
Regulations (CFR) Title 45, part164; 3) Applicable local regulatory guidelines

\subsection{Inclusion/Exclusion Criteria}

Each subject was required to have a closed single traumatic cervical spinal cord injury occurring within 12 hours of first dosing. Included in the subject population were male or female cervical AIS A subjects $\geq 16$ to $\leq 80$ years and male or female cervical AIS B or C subjects $\geq 16$ to $\leq 70$ years. Subjects were randomly assigned to either Placebo or active treatment in a 1:1 ratio within each of the following 5 strata based on their baseline AIS injury level as follows:

a) AIS A with a level of injury at either C4, C5, C6, or C7 (for $\mathrm{C} 4$, the subject must have had at least 1 point of motor activity within the zone of partial preservation (ZPP) inclusive of $\mathrm{C} 5$ to thoracic level 1 [T1]). In addition, an AIS A subject may be included if ALL of the following are present 1) the most caudal intact sensory segment (both pinprick and light touch) was $\mathrm{C} 3,2$ ) at least one side (right or left) had both intact pinprick and light touch sensation in the $\mathrm{C} 4$ dermatome, AND 3) at least 1 point of motor activity within the ZPP inclusive of $\mathrm{C} 5$ to $\mathrm{T} 1$.

b) AIS B or $\mathrm{C}$ with a neurological level of injury at either $\mathrm{C} 3, \mathrm{C} 4, \mathrm{C} 5, \mathrm{C} 6, \mathrm{C} 7$, or $\mathrm{C} 8$.

Subjects were excluded if they were 1) unable to sign informed consent, 2) unable to participate in the ISNCSCI examination, 3) unable to discontinue specific classes of medications (potent cytochrome P450 (CYP) 3A4/5 inducers and/or use of potent $\mathrm{P}$ glycoprotein (P gp) inhibitors) 4) had renal or hepatic compromise, 5) were pregnant or breastfeeding or 6) had any other medical condition that precluded treatment in the study in the investigator's opinion.

\subsection{Study Conduct}

The study was conducted initially at an acute care hospital and could continue to a rehabilitation or spine center facility. Additionally, follow-up visits could be completed on an outpatient basis. Informed consent was obtained from the subject (assent was obtained from subjects younger than 18 years) or the subject's Legally Authorized Representative (LAR) before any study procedures, including screening procedures and pre-dose baseline evaluations, were conducted. Administration of the first dose of study drug must occur within 12 hours of injury. In order to standardize dosing time, at least 8 hours but no more than approximately 24 hours elapsed between the first and second doses of study drug, between subsequent doses of study drug, or between any missed doses of study drug for a given subject. Daily dosing with study drug was standardized to occur, preferably, at 9:00 $\mathrm{AM}$ ( \pm 1 hour) for each subject after he/she had received 1 or 2 doses of study drug. For each subject, injections of study drug were administered once daily (after standardization) by IV bolus injection, which was commonly known as 'IV push.' A peripheral IV catheter or midline catheter (a midline catheter was shorter in length than a 
peripherally inserted central catheter [PICC] and would not reach the heart directly) may be inserted before dosing for administration of study drug. A central line or PICC could be used for administration of study drug provided that the port used for drug administration emptied peripherally and not directly into the heart. The study drug administration period was 27 to 28 days with 7 to 28 total doses of study drug and 22 weeks of post-dose follow up (26 weeks total study duration per subject). No subject received more than 28 doses of study drug. At selected sites, PK of SUN13837 and its metabolite, 1 benzyl $\mathrm{N}$ methylpiperidin 4 amine (BMP
[ASB15490]) were assessed using both rich (intensive) and sparse sampling techniques.

After dosing was completed, the study continued with study related efficacy and safety assessments. This study included 7 study visits over the course of 26 weeks beginning with a Screening Period (within 12 hours of injury) that also included pre-dose baseline evaluations (immediately after screening, within 12 hours after injury). Additional interim visits for safety could be conducted during the study at the discretion of the investigator or the Sponsor. The last safety assessments were conducted on Day 182 ( \pm 7 days). (Figure 1)

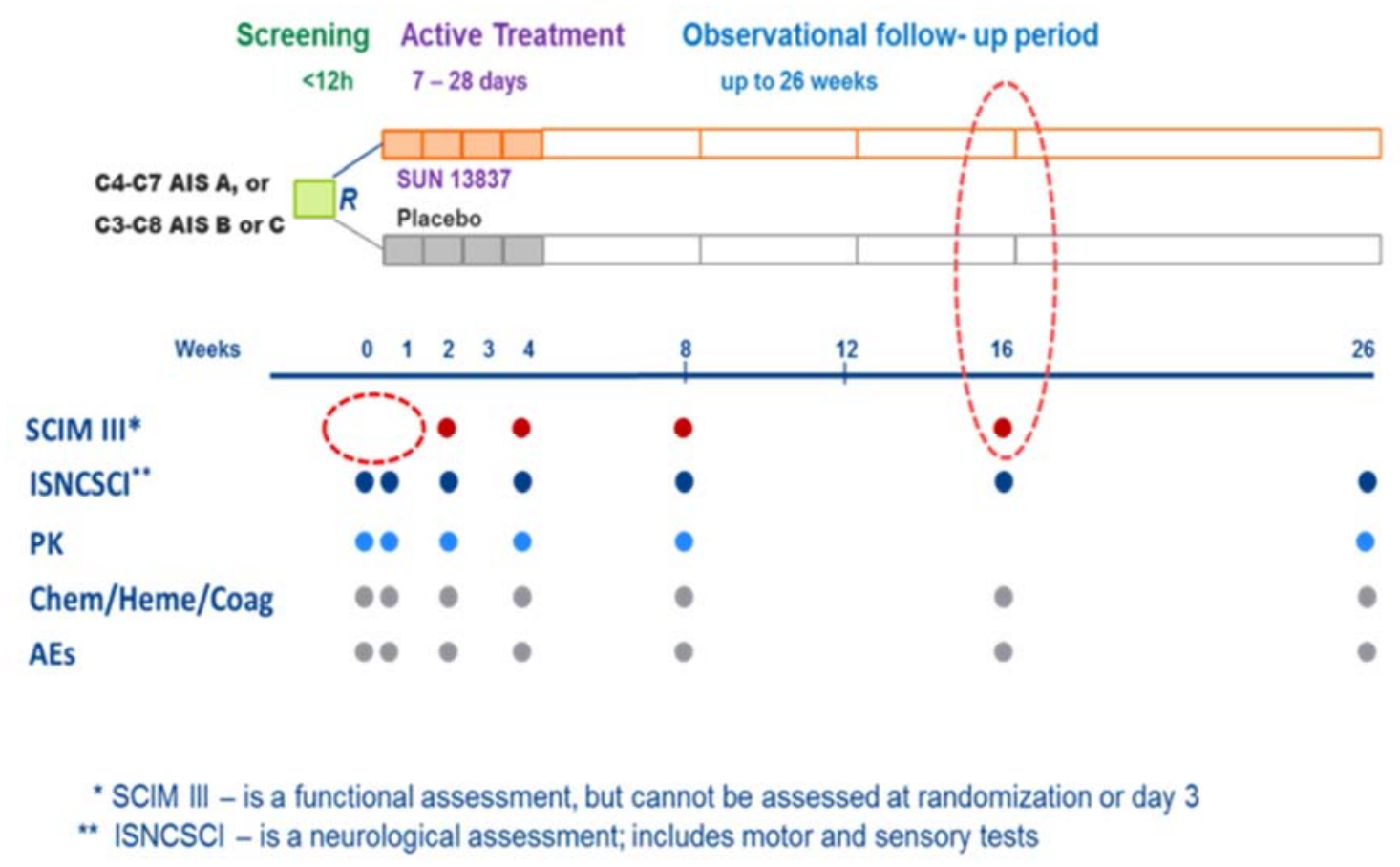

Figure 1. Efficacy and safety assessments.

Subjects were monitored throughout the study for adverse reactions to the study drug and/or procedures. Subjects were instructed to inform the appropriate study personnel of any AEs that may occur at any time during the study. Subjects were monitored by appropriate study personnel for treatment emergent adverse events (TEAE), new or worsening of preexisting conditions.

\subsection{Assessments}

The total SCIM III score was chosen for primary endpoint assessment because it was a reliable measure of function and relates to the ISNCSCI which was based on neurological evaluation. In this study, a pre-dose or baseline SCIM III was not obtained as evaluation of functional measures is difficult and unreliable during the immediate 12-hour, post-injury period. Therefore, the first measure of SCIM III was performed two weeks after injury. The Week 16 data was chosen for primary end-point evaluation because the majority of recovery that occurs after ASCI will be found within this time period (Steeves et al., 2007).

The secondary endpoint of total motor score (TMS) was chosen to determine if the change in SCIM III was consistent with neurological recovery. However, caution in comparison of these two measures should be maintained as changes in respiratory and sphincter management could have a significant impact on overall SCIM III as related to subject medical status change or lifestyle choice and have little to do with function.

The Intent-to-Treat (ITT) Population, the primary population for efficacy, consisted of all randomized subjects who had at least 1 SCIM III evaluation and received at least 7 (partial or complete) IV bolus dose injections of the study drug in SUN13837 group or in Placebo group. All primary, secondary and exploratory endpoints were analyzed using ITT population. Population size was calculated based on the natural history of the total SCIM III using data from the ISNCSCI and SCIM III scores obtained from the European Multicenter Spinal Cord Injury database. Based on a recent publication (Scivoletto et al., 2013), the sample size was based on the primary endpoint of total SCIM III score at Day $112( \pm 7$ days). Under placebo treatment, the mean SCIM III score at Day 112 ( \pm 7 days) was expected to be 27 with an SD of 14.6.

\subsection{Statistics}

A sample size of 28 subjects (who completed 7 doses per 
subject) per arm was required to show a statistically significant difference of 10 points in the total SCIM III at Day 112 ( \pm 7 days) using a 2-sample t-test at a 2-sided type I error of 0.10 and power of $80 \%$.

\subsection{Study Oversight}

The study was conducted in accordance with current Good Clinical Practice. The protocol and all amendments were approved by local Ethics Committees and all subjects consented to study participation. Due to the nature of the injury, where signature was not possible, a Legally Authorized Representative could sign once the subject acknowledged willingness to participate.

A single independent data monitoring committee (DMC) was established to monitor safety on an ongoing basis throughout the study. There was no interim analysis for efficacy.

The DMC consisted of 2 physicians and 1 biostatistician. The DMC reviewed unblinded safety data. Every month the DMC received all SAEs reported on a Serious Adverse Event Form from the prior month. They were asked to evaluate any findings during and at study completion that were 1) atypical of ASCI or its sequelae or 2) related to SUN13837.

The study was sponsored by Daiichi-Sankyo Pharmaceuticals. All authors were involved with the collection, analysis, or interpretation of the data. The authors vouch for the accuracy and completeness of the data and analysis and for the fidelity of the study to the protocol.

\section{Results}

About 1400 subjects with any type of cervical injury were pre-screened. After initial assessment in the Emergency Department, 69 subjects were screened as eligible to participate based on inclusion and exclusion criteria. Of the 69 subjects screened in the United States, Czech Republic, United Kingdom, France and Spain, 65 subjects were randomized. The United States contributed 57 subjects, France and the UK, 3 subjects each and the Czech Republic and Spain, 1 subject each

Two subjects were given approval to receive drug one hour outside the 12-hour administration window. Four of these subjects were not randomized and not treated due to unwillingness to consent. Four of the subjects were randomized but not treated. 61 subjects were included in the safety population and 55 in the ITT population.

Of the 61 subjects who received study drug, 57 received at least 7 doses. On average, SUN13837 subjects received 21.6 injections while Placebo subjects received 22.3 injections. Of the 61 subjects, $59 \%$ received all 28 injections; $56.7 \%$ of SUN13837 subjects vs $61.3 \%$ of Placebo subjects. A total of 37 subjects completed the treatment period, ie, received all 28 doses. Reasons for early withdrawal from study treatment included 'other' $(\mathrm{n}=10)$, AEs $(\mathrm{n}=8)$, withdrawal of consent $(\mathrm{n}=5)$ and Investigator's decision $(\mathrm{n}=1)$. The distribution of subjects may be seen in Figure 2.

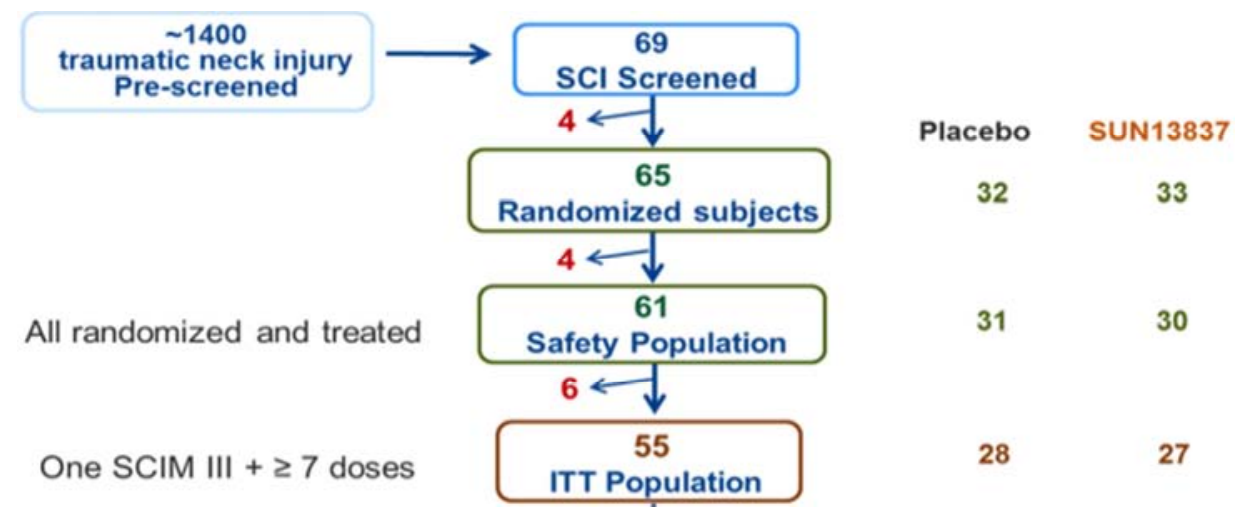

Figure 2. Subject Distribution.

The majority of subjects were Male $(85.2 \%)$, Caucasian $(63.9 \%)$, not of Hispanic or Latino ethnicity $(90.2 \%)$ and less than 55 years old $(82.0 \%)$. The average age and weight of the subject population was 38.4 years and $84.3 \mathrm{~kg}$, respectively. There were no significant imbalances between the two treatment groups with respect to the demographic variables.

In the majority of subjects, the cause of spinal cord trauma was due to falls $(44.3 \%)$ followed by motor vehicle accident $(29.5 \%)$ and sports/recreation injuries $(23.0 \%)$. No concomitant injuries were reported in $49.2 \%$ of subjects.

A summary of all results may be found in Table 1 . The overall total mean ISNCSCI score at Baseline, inclusive of both motor and sensory scores was slightly higher for the
SUN13837 group compared to Placebo (76.2 vs 69.8, respectively).

The LS Means of the total SCIM III score seen in this tetraplegic population show a very large degree of disability among the SUN13837- and placebo-treated subjects at Week 2 (15.87 vs. 10.26, respectively) (Figure 3). By Week 16 post-injury there remains among SUN13837 and placebotreated subjects less than half the functional ability of a healthy spinal cord ( 38.14 vs. 33.60 , respectively). The mean Total SCIM III score difference between the 2 treatment groups at Week 16 was $4.54(\mathrm{SE}=6.524)$, which was not statistically significant $(\mathrm{p}=0.4912)$. Therefore, the primary end-point was not reached. Overall, larger effects were observed in AIS C6-C7 and AIS B and C strata as compared with AIS A C4-C5 (see Table 2) 


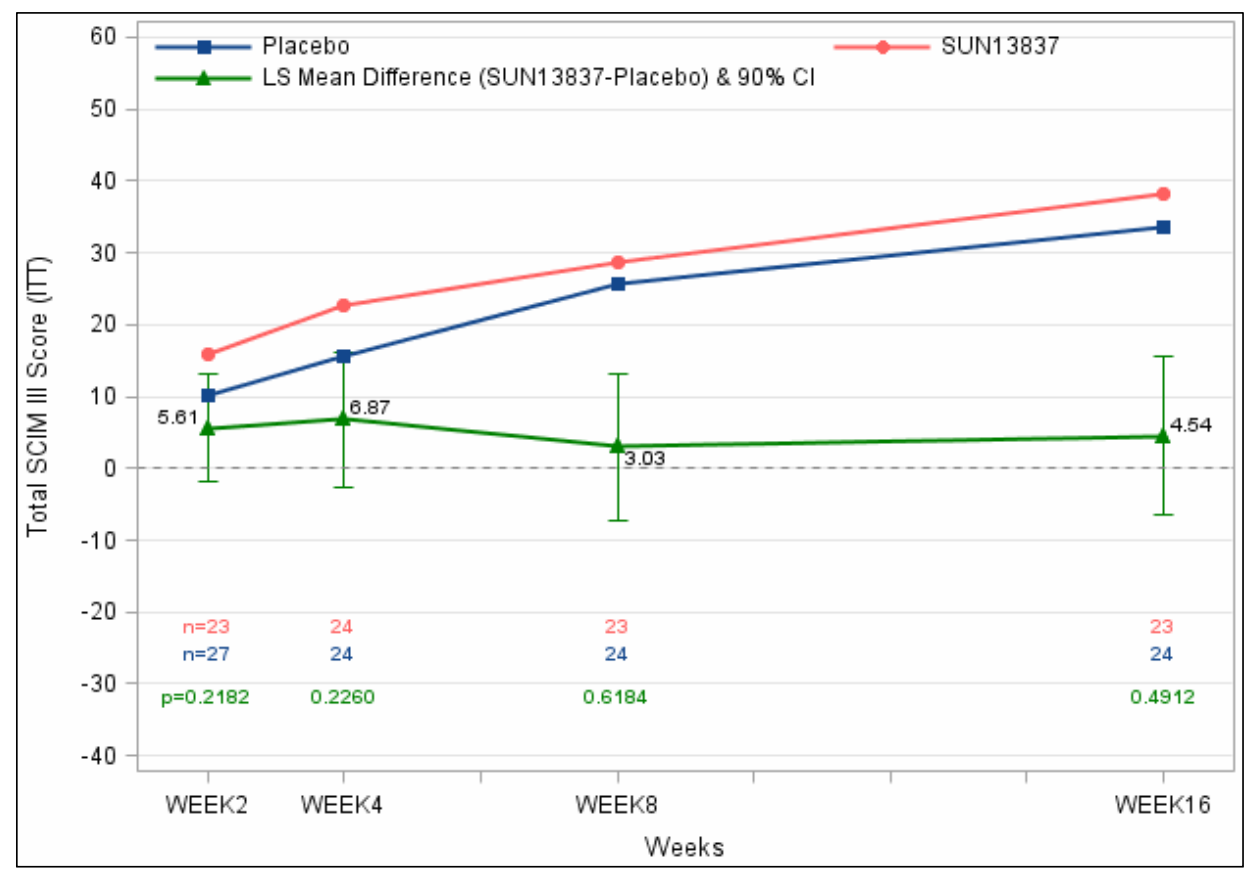

Figure 3. Total SCIM III Scores.

Table 1. Summary of results.

\begin{tabular}{llllc}
\hline Parameter at Week 16 or & LS Mean (SE) & & \multicolumn{1}{c}{ p-value } \\
\hline Final Assessment ( ITT ) & SUN13837 & Placebo & \multicolumn{2}{l}{ Difference or Odds Ratio } \\
Total SCIM III & $38.14(4.70)$ & $33.60(4.70)$ & $4.54(6.52)$ & 0.4912 \\
Secondary Efficacy Variable & & & & \\
Total Motor Score of ISNCSCI (change from baseline) & $16.58(4.28)$ & $14.07(4.18)$ & $2.51(5.87)$ & 0.6719 \\
Combined Self-Care and Mobility Score of SCIM III & $18.73(3.12)$ & $15.02(3.10)$ & $3.71(4.30)$ & 0.3951 \\
UEMS of ISNCSCI (change from baseline) & $9.92(1.69)$ & $4.95(1.67)$ & $4.97(2.30)$ & 0.0351 \\
Lower Extremity Motor Score (LEMS) of ISNCSCI (change from baseline) & $6.13(2.57)$ & $9.96(2.52)$ & $-3.83(3.58)$ & 0.2906 \\
\hline
\end{tabular}

Table 2. Total SCIM III Score by AIS Stratum Level \& Treatment Group (ITT).

\begin{tabular}{|c|c|c|c|c|c|c|}
\hline \multirow{3}{*}{ Visit } & \multicolumn{6}{|l|}{ Mean (SD) } \\
\hline & \multicolumn{2}{|l|}{ AIS A C4-C5 } & \multicolumn{2}{|l|}{ AIS A C6-C7 } & \multicolumn{2}{|l|}{ AIS B or C } \\
\hline & SUN13837 (N = 15) & Placebo $(\mathrm{N}=13)$ & SUN13837 $(\mathbf{N}=\mathbf{5})$ & Placebo $(\mathrm{N}=6)$ & SUN13837 $(\mathbf{N}=7)$ & Placebo $(\mathrm{N}=9)$ \\
\hline Week 2 & $7.5(5.57)$ & $3.5(4.35)$ & $6.0(6.00)$ & $5.0(5.51)$ & $35.7(30.79)$ & $25.4(31.66)$ \\
\hline Week 4 & $10.5(7.74)$ & $8.5(8.02)$ & $16.3(15.63)$ & $8.8(8.28)$ & $51.2(38.00)$ & $22.6(12.23)$ \\
\hline Week 8 & $14.4(9.86)$ & $20.0(17.12)$ & $22.0(16.47)$ & $17.2(6.49)$ & $71.0(33.19)$ & $33.6(10.36)$ \\
\hline Week 16 & $24.8(12.75)$ & $26.8(25.80)$ & $40.8(19.52)$ & $25.5(5.75)$ & $67.3(34.36)$ & $42.9(16.61)$ \\
\hline
\end{tabular}

For the combination of Self-Care and Mobility subscale scores, by Week 16 the LS Mean (SE) for SUN13837 vs Placebo was 18.73 (3.124) vs 15.02 (3.101); slightly better in the active group. The LS Mean (SE) difference between the 2 treatment groups was 3.71 (4.298) which was not statistically significant with $\mathrm{p}$-value $=0.3951$.

Average Self-Care subscale scores for all strata were similar between groups at the first measurement, Week 2 with a mean (SD) of 1.6 (3.24) observed for SUN13837 and 1.4 (4.06) for Placebo. By Week 16 a slight improvement was seen in the SUN13837 group compared to Placebo; mean (SD) of 8.2 (6.54) vs 5.8 (5.72), respectively. At Week 16, no statistically significant difference between the 2 treatments was observed $(p$-value $=0.421)$.

A comparison of TMS from baseline to the Day $112( \pm 7$ days) (Week 16 or final) assessment was performed for
Placebo- and SUN13837-treated subjects in the ITT population. By Week 16, the LS Mean (SE) of the TMS for SUN13837 was only slightly better than Placebo, 16.58 (4.277) vs 14.07 (4.175) in SUN13837. The difference between the 2 treatment groups was 2.51 (5.874) which was not statistically significant with p-value $=0.6719$.

At baseline, the average UEMS in SUN13837-treated subjects was 3.6 points lower than that of Placebo-treated subjects. Steady improvements in UEMS were observed in SUN13837-treated subjects at every visit beginning with Day $3($ LS Mean $=2.08)$ to Week $16($ LS Mean $=9.92)$. Improvements in UEMS were also seen in the Placebotreated subjects but were smaller in magnitude beginning at Week 2. The LS Mean difference between SUN13837 and Placebo, favoring SUN13837 were statistically significant at every visit ( $p$-value $<0.10$ ). By Week 16, the LS Mean (SE) 
change from baseline in UEMS scores was 9.92 in SUN13837-treated subjects compared to 4.95 in Placebotreated subjects ( $\mathrm{p}$-value $=0.0347$ ).

A significant treatment effect in favor of SUN13837 was observed from Day 3 onwards (p-value $<0.10$ ). At Week 16, a treatment effect of 4.97 was seen. In this analysis at Week 16 by AIS strata, the largest treatment difference was seen in the AIS B and C strata in which SUN13837-treated subjects had an average change from baseline in UEMS of 25.40 compared to 6.86 in Placebo-treated subjects. As a result, the AIS B and C stratum may have contributed heavily to the overall treatment effect with lesser contribution by AIS A C4-C5 and C6-C7 strata (Table 3).

Table 3. UEMS at Week 16 - Stratum Analysis (ITT).

\begin{tabular}{lllll}
\hline AIS Strata & Mean (SD) & & & Change from Baseline \\
\hline & Observed at Week 16 & & SUN13837 & Placebo \\
\hline & SUN13837 & Placebo & $7.00(5.689)$ & $5.30(7.959)$ \\
AIS A C4-C5 & $18.2(12.47)$ & $15.4(11.72)$ & $2.50(7.141)$ & $0.33(3.386)$ \\
AIS A C6-C7 & $22.3(7.41)$ & $28.5(11.98)$ & $25.40(13.259)$ & $6.86(10.574)$ \\
AIS B and C & $41.8(12.77)$ & $29.0(13.70)$ & \\
\hline
\end{tabular}

Safety evaluations were performed by an Independent Data Monitoring Committee who concluded that no safety signals were evident in this small study.

Pharmacokinetic evaluation did not show any relationship between blood levels and drug effect in any of the measurements evaluated. However, 6 subjects of 18 had blood levels above the predetermined modeled NOAEL concentrations based on toxicological guidance from nonclinical data. Five of these subjects had elevated Cmax, ss and 2 had elevated AUCss (0-24) (one subject had both elevated Cmax, ss and AUCss (0-24)). This indicates a need for further evaluation of dose amount if any more clinical work is to be done with SUN13837.

\section{Discussion}

The purpose of this study was to compare the efficacy, safety and pharmacokinetics of once-daily intravenous administration of SUN13837 to Placebo in the treatment of acute spinal cord injury. The final population of subjects included complete (AIS A) and incomplete (AIS B and C) subjects where complete and incomplete injury is defined by the International Standards for Neurological Classification of Spinal Cord Injury $[8,9]$. AIS A subjects had to have an injury between $\mathrm{C} 4$ and $\mathrm{C} 7$ inclusive (C3 subjects were enrolled if there was a motor level of $\mathrm{C} 4$ on 1 side) and AIS $\mathrm{B}$ and $\mathrm{C}$ subjects had to have their injury between $\mathrm{C} 3-\mathrm{C} 8$ inclusive as diagnosed within 12 hours.

A total of 69 subjects were screened of whom 65 were randomized. The safety population of 61 subjects included all individuals who received at least one dose of study drug. The ITT population of 55 subjects had at least 1 SCIM III evaluation and least 7 doses of study drug. The majority of subjects, $59 \%$, received 28 doses of study drug. The original design of the study was to have each subject receive 28 doses of study drug. This was changed during study conduct as a result of the ongoing nonclinical program that demonstrated as few as seven doses would be adequate for efficacy (Data on File, Daiichi Sankyo, Inc.). For analysis purposes, the data were reduced to three strata of AIS A, C4 - C5, AIS A C6 C7, and AIS B and C.

Data from one small meta-analysis indicated that a clinically meaningful change in the primary endpoint of the total SCIM III score among all strata could be defined as a10-point difference between the 2 groups at Week 16 that would also be statistically significant [10]. However, this definition of 'clinically meaningful' is controversial because such a difference depends on the AIS Grade and level of injury [11]. In this study, assessment of the primary endpoint of the total SCIM III did not meet the pre-defined criteria for efficacy. A non-statistically significant treatment difference in the primary outcome measure of 4.5 points favoring SUN13837 was shown at Week 16.

At baseline, the total mean score for the ISNCSCI was 6.4 points higher in the SUN13837 treatment group compared to Placebo. This difference in pre-dose total ISNCSCI indicates that the SUN13837 group began the study with a better neurological baseline and therefore would be expected to have a slightly better overall outcome. The small post-dose improvements that were seen for SUN13837 in the majority of the parameters of ISNCSCI and SCIM III may have contributed to this difference. Therefore, any truly meaningful clinical improvement must account for this baseline difference in total ISNCSCI that favored SUN13837.

Unlike the total SCIM III score that differed at Week 2 (the first measurement obtained), the SCIM III Self-Care data were the same between groups at this time. The Self-Care subscale for all strata combined is similar at Week 2, indicating a similar baseline and resulted in a slight numerical treatment effect by Week 16 (1.46). However, this result is not statistically significant.

Treatment effects on total SCIM III score were very different in the 3 AIS strata. While the largest stratum, the AIS A C4 - C5 subjects demonstrated a small favorable response to Placebo ( 2 points), the AIS A C6 - C7 and more notably the AIS B and $\mathrm{C}$ subjects showed large differences between the 2 treatment means of 15.3 and 24.4 points at Week 16, favoring SUN13837. No hypothesis testing was done in these subsets of subjects as the numbers of subjects in each stratum were too small for a statistical comparison. In the incomplete-injured stratum (AIS B and C), there were a larger number of $\mathrm{C}$ subjects in the SUN13837 group compared to the Placebo group (5 vs 2 , respectively). This 
may explain the larger treatment effect seen, which is important because AIS C subjects typically have a greater degree of natural recovery than AIS B subjects [12].

Because of the large difference in the distribution of subjects in the incomplete stratum of AIS B and C, we tested the assumption that there was a treatment effect by evaluation of the AIS A stratum with ZPP. The incomplete-injured subject and the AIS A stratum with ZPP share the common attribute of having clinical evidence of partially injuredtissue that could undergo recovery [13]. In this situation, the effect on partial injury may have some degree of similarity to the AIS A with ZPP and the AIS B and C but the analysis did not confirm this assumption. It is likely that the larger number of C subjects in the SUN13837 AIS B and C stratum had significant impact on the larger observed numerical effect in the treatment group due to the greater motor response seen in these 6 subjects [14]. Therefore, treatment was not an important factor in the SUN13837 effect seen in the AIS B and C stratum.

The secondary endpoint of TMS failed to show any statistically significant or clinically meaningful difference. This would be expected as the majority of subjects were AIS A or complete-injured subjects. These subjects are least likely to gain recovery in motor points [12].

Combination of SCIM Self-Care and Mobility subscales was indicated due to the inclusion of both AIS A (complete injury) and AIS B and C (incomplete injury). In this situation, the incomplete-injured subject is more likely to have mobility in the lower limbs such that combination of these subscales is warranted [15]. Once again results showed no statistical difference between the treatments and only a small numerical improvement of 3.7 points in the SUN13837 group compared to Placebo.

Consistent with the primary endpoint, none of the SCIM III subscales (Self-Care, Self-Care and Mobility combined, and Respiration and Sphincter Management) showed any statistically significant or clinically relevant treatment effects.

The exploratory analysis of UEMS among all strata combined showed a statistically significant treatment difference at Day 3 and beyond. This is of particular importance as the UEMS is the ISNCSCI score most closely associated with cervical injury recovery.

As a cautionary note however, the analysis by AIS strata showed a notable inconsistency of effect across the 3 strata with larger effect in the AIS B and C stratum. While an expected positive correlation between UEMS and SCIM III Self-Care subscale was seen in the study, this was not consistent across the 3 AIS strata. In addition, the SCIM III self-care data, which is highly correlated to the UEMS [16], failed to show any clinically meaningful improvement.

\section{Conclusion}

The overall conclusion of this study is that SUN13837 shows some evidence for a small improvement in neurological recovery among parameters in the various strata evaluated, but the small improvement did not reach a level considered to be clinically meaningful with regard to effect on function. No safety concerns were noted. The dose may need to be lowered if any further clinical studies are performed with this drug in acute spinal cord injury.

\section{Acknowledgements}

The ASCENT-ASCI (Asubio Spinal Cord Early Neurorecovery Treatment for Acute Spinal Cord Injury) Study Group was comprised of 29 investigators over 5 countries. Daiichi-Sankyo gratefully thanks the Physicians, Nurses, other study personnel, and, most importantly, the patients who consented to participate in this study.

Dr. Armin Curt for his cooperation in the use of the European Multicenter Spinal Cord Injury Database

Dr. John Steeves for his clinical and statistical to the analyses

Support: Daiichi Sankyo Pharma Development, Basking Ridge, NJ

\section{References}

[1] Strauss DJ, De Vivo MJ, Paculdo DR, Shavelle RM. Trends in life expectancy after spinal cord injury. Arch Phys Med Rehabil. 2006 Aug; 87 (8): 1079-85.

[2] Wyndaele M, Wyndaele, JJ. Incidence, prevalence and epidemiology of spinal cord injury: what learns a worldwide literature survey? Spinal Cord. 2006 Sep; 44 (9): 523-9.

[3] Berkowitz M. The costs of spinal cord injury. In: Lin V, ed in chief; Cardenas D, Cutter N, Frost F, Hammond M, Lindblom LB, Perkash I, et al, eds. Spinal Cord Medicine Principles and Practice. New York, NY: Demos Medical Publishing, Inc.; 2003:949-55.

[4] Bosshart HT. Social issues in spinal cord injury. In: Lin V, edin chief; Cardenas D, Cutter N, Frost F, Hammond M, Lindblom LB, Perkash I, et al, eds. Spinal Cord Medicine Principles and Practice. New York, NY: Demos Medical Publishing, Inc.; 2003:941-8.

[5] Rabchevsky AG, Fugaccia I, Turner AF, Blades DA, Mattson MP, Scheff SW. Basic fibroblast growth factor (bFGF) enhances functional recovery following severe spinal cord injury to the rat. Exp Neurol. 2000 Aug; 164 (2): 280-91.

[6] Song BW, Vinters HV, Wu D, and Pardridge WM. Enhanced neuroprotective effects of basic fibroblast growth ractor in regional brain ischemia after conjugation to a blood brain barrier delivery vector. J Pharmacol Exp Ther. 2002 May; 301 (2): 605-10.

[7] Bossard C, Laurell H, Van den Berghe L, Meunier S, Zanibellato C, Prats H. Translokin is an intracellular mediator of FGF 2 trafficking. Nat Cell Biol. 2003 May; 5 (5): 4339.

[8] Steeves JD, Lammertse D, Curt A, Fawcett JW, Tuszynski $\mathrm{MH}$, Ditunno JF, et al. Guidelines for the conduct of clinical trials for spinal cord injury (SCI) as developed by the ICCP panel: clinical trial outcome measures. Spinal Cord. 2007 Mar; 45 (3): 206-21. Epub 2006 Dec 19.

[9] http://www.asia-spinalinjury.org/elearning/ISNCSCI.php. 
[10] Scivoletto G, Tamburella F, Laurenza L, Molinari M. The spinal cord independence measure: how much change is clinically significant for spinal cord injury subjects. Disabil Rehabil, 2013 Oct; 35 (21): 1808-13.

[11] Lammertse D, et al., oral communication, Feb 2016.

[12] Fawcett JW, Curt A, Steeves JD, Coleman WP, Tuszynski $\mathrm{MH}$, Lammertse D, et al. Guidelines for the conduct of clinical trials for spinal cord injury as developed by the ICCP panel: spontaneous recovery after spinal cord injury and statistical power needed for therapeutic clinical trials. Spinal Cord. 2007 Mar; 45 (3): 190-205. Epub 2006 Dec.

[13] Report from the Institute of Medicine: Spinal cord injury: progress, promise, and priorities / Committee on Spinal Cord Injury, Board on Neuroscience and Behavioral Health; Catharyn T. Liverman, et al., Editors. National Academies
Press, 500 Fifth Street, N. W., Lockbox 285, Washington, DC 20055; 2005 p 32.

[14] Kiwerski J. The natural history of neurological recovery in patients with traumatic tetraplegia Paraplegia. 1989 Feb; 27 (1): $41-5$.

[15] McKinley W, Pai AB, and Kulkarni U. Functional Outcomes per Level of Spinal Cord Injury. 2015-Jul-15. Retrieved from: http://emedicine.medscape.com/article/322604-overview.

[16] Rudhe C, van Hedel HJ. Upper extremity function in persons with tetraplegia: relationships between strength, capacity, and the spinal cord independence measure. Neurorehabil Neural Repair. 2009 Jun; 23 (5): 413-21. 